\title{
Neuropathic Pain: Literature Review and Recommendations of Potential Phytotherapies
}

\author{
Karim Raafat*, Rawan El-Haj, Dania Shoumar, Rana Alaaeddine, Yousra Fakhro, Natalie Tawil, Farah Shaer, Amani \\ Daher, Natalie Awada, Ali Sabra, Khouloud Atwi, Malak Khaled, Raneem Messi, Nour Abouzaher, Mohamed \\ Houri and Samer Al Jallad
}

Karim Raafat*, Rawan El-Haj, Dania Shoumar, Rana Alaaeddine, Yousra Fakhro, Natalie Tawil, Farah Shaer, Amani Daher, Natalie Awada, Ali Sabra, Khouloud Atwi, Malak Khaled, Raneem Messi, Nour Abouzaher, Mohamed Houri and Samer Al Jallad

Department of Pharmaceutical Sciences, Faculty of Pharmacy, Beirut Arab University, 115020 Beirut, LEBANON,

\section{Correspondence}

\section{Karim Raafat,}

Department of Pharmaceutical Sciences, Faculty of Pharmacy, Beirut Arab University (BAU), 115020 Beirut, LEBANON.

Phone number:+961 1300110:

Fax: +961 1300110 Ext:2599

E-mail: k.raafat@bau.edu.lb, karim.raafat@yahoo.com

History

- Submission Date: 19-02-2017;

- Review completed: 12-04-2017;

- Accepted Date: 03-05-2017

\section{DOI : 10.5530/pj.2017.4.72}

Article Available online

http://www.phcogj.com/v9/i4

\section{Copyright}

(C) 2017 Phcog.Net. This is an openaccess article distributed under the terms of the Creative Commons Attribution 4.0 International license.

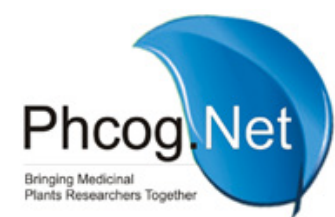

\begin{abstract}
Introduction: One of the common syndromes that affect humanity is neuropathic pain (NP), yet poorly understood with no efficient treatment till now. Consequently, it is extremely crucial to seek further evidence for accurate diagnosis and optimum treatment. The aim of this work is to summarize the findings related to neuropathic pain in terms of etiology, types, diagnosis, current and future management prospective, and identify the gaps in neuropathic pain therapy. Methods: A literature investigation was carried out by analyzing classical peer reviewed papers and textbooks, taking into consideration worldwide well established scientific databases mainly PUBMED and SCOPUS to retrieve accessible published literature. The selection of phytotherapies was based upon their potentials in relieving NP in pre-clinical or clinical models. Results: One of the most critical points in this research is that recommendations of the future plans should be focused on the engagement of phytotherapy in the treatment regimen aiming at improving patients' quality of life and reaching optimum efficacy and minimum toxicity. Phytotherapies offer relatively low-risk options to NP patients and have an increasing evidence to be the future of neuropathic pain management. Patients suffering from neuropathy are depending increasingly on phytotherapies; however, they need more clinical trials in order to fully understand their mechanism of actions. Conclusion: Health-care specialists should be regularly informed about neuropathic pain current therapies and promising future phytotherapies, bearing in mind the risk/benefit profile of the utilization of these therapies in the amelioration of NP.

Key words: Neuropathy Pain, Current therapies, Diagnosis, Mechanisms, Recommendations, Phytotherapies.
\end{abstract}

\section{INTRODUCTION}

Neuropathic pain (NP) is now a global health concern without adequate therapeutic approach. NP causes difficulties in daily activities, decreases performance and increases health care costs. Pain grading in NP is a crucial method for diagnosing NP. ${ }^{1}$

\section{Pain grading}

NP is a syndrome and not a disease. It might be induced by a disease or a lesion of the peripheralor central-nervous system (CNS). The reason of NP might be either due to an infection, injury or metabolic disorder, or unknown cause. The same lesion or disease may induce the development of neuropathic, somatic or even psychogenic pain. ${ }^{2}$

The four criteria for grading NP diagnosis are as follows: I. Neuro-anatomical related pain.

II. There is a related lesion or disease in the history of nervous system.

III. The existence of positive or negative neurologic manifestations with the abundance of pain in neurological assessment or in the more objective confirmatory tests (quantitative sensory testing, laboratory tests).

IV. The existence of related disease or lesion by no less than a single confirmatory test.
Consistent with this criteria NP can be classified into "defined" (all criteria present), "probable" (I and II, in addition either III or IV) or "possible" (I and II, with no confirmatory tests). ${ }^{3}$

Moreover, risk factors and epidemiology are cornerstones in understanding NP.

\section{Risk factors and Epidemiology}

The literature survey has shown fewer studies on the NP epidemiology. A study on patients evidenced neuropathic pain in $8.2 \%$ of adult patients having significantly higher pain intensity than others in a British study. ${ }^{1,4}$ Moreover, in a randomly selected adult population using the Douleur Neuropathique four Questions, neuropathic pain were identified in $6.9 \%$ of the examined persons in a French study. ${ }^{5}$ NP risk factors includes: females, elderly age, minor level of education, living in a rural area worse health (compromised physical, mental and social wellbeing) ${ }^{5-9}$

\section{Pathomechanisms}

Pathomechanisms causing the development of NP are divided into 3 groups: first, electrophysiological properties of the cellular membrane of the first 
sensory neuron changes, which include changes in nociceptor excitability, gene expression changes in neuronal cell body and in the release of neurotransmitters. Second, the alteration in impulse-processing in the spinal-cord dorsal-horns. Third, Disorders in the CNS such as disturbed balance between the activity of ascending excitatory systems and descending inhibitory systems. Moreover, the autonomic nervous system dysfunction might be also entangled with the NP development. ${ }^{1,}$

\section{Screening}

The screening scales can be used in identifying NP or the presence of a clear neuropathic factor in the pain syndrome. They may be used if medical history or physical examination reveals typical signs of neuropathic pain. The most widely used screening scales include: "Douleur Neuropathique 4 Questions" (DN4), "Leeds Assessment of Neuropathic Symptoms and Signs" (LANSS), NP questionnaire (NPQ) and NP DETECT. ${ }^{1}$

\section{Diagnosis and Current Therapies of Neuropathic Pain Postherpetic Neuralgia (PHN)}

Diagnosis of PHN may be in patients complaining of severe unilateral pain in dermatomes where herpes zoster virus has caused lesions. This diagnosis is possible after the lesions have completely healed. ${ }^{11,12}$

Opioids, amitriptyline, 5\% lidocaine, gabapentin, pregabalin, and $8 \%$ capsaicin are the most efficatious first-line agents in treatment of PHN. Type and intensity of pain decide which of these agents is to be initiated.

Recommendations suggest that monotherapy should be started. Switching to a combination is only recommended in case of failure of monotherapy. ${ }^{12,13}$

\section{Complex Regional Pain Syndrome (CRPS)}

Diagnosis of CRPS should be performed by a specialist in neuropathy, which is a type of chronic pain affecting limbs. ${ }^{14}$

It is possible to give bisphosphonates, antispastic drugs, or invasive procedures in case of failure of medications of neuropathic pain.

As experts say, an early multidirectional is found to be the most effective treatment, where physical therapy is a principle component of this therapy. Psychological therapy is also included in the treatment regimen if the pain is psychological in nature. ${ }^{1,15-18}$

\section{Diabetic Polyneuropathy}

Diabetic polyneuropathy is caused by both types of diabetes mellitus (DM). Prevention of neuropathy in DM patients is achieved through maintaining a proper level of glucose in blood. ${ }^{19,20}$

As for treatment, gabapentin, pregabalin, TCAs, venlafaxine ER and duloxetine are the first-line agents to treat diabetic neuropathy, while opioids and tramadol are the second-line agents.

Primarily patients are given a single first-line drug. Switching to a different drug from an alternate therapeutic group is done in case of failure of the first-line agent. Knowing that alleviation of pain is not achieved, we consider one of the following approaches: choose a secondline drug; choose a synthetic or natural drugs having other mechanism of actions; or use drug combinations. ${ }^{21-24}$

\section{Trigeminal Neuralgia (TN)}

$\mathrm{TN}$ is diagnosed in patients who experience chronic NP in the trigeminal nerve area. ${ }^{25,26}$ The first-line agent for treatment of TN is carbamazepine. However, if carbamazepine is contraindicated, then the use of clonazepm, baclofen, or antiepileptics (gabapentin, lamotrigine or pregabalin) is indicated. Surgery is recommended in case of failure of pharmacotherapy, yet treatment with botulin toxin is advisable prior to a surgery. ${ }^{27-30}$

\section{Post-amputation Pain}

Phantom pain was incident in amputated patients with extremity and stump pain located surrounding the post-surgical stump scar. ${ }^{31}$

Due to the fact that strong opiods, tramadol, gabapentin, lidocaine infusions, calcitonin pregabalin or amitriptyline are recommended, evidence of effectiveness of these therapeutic agents is lacking. Other types of treatment have emerged, such as psycotherapy and physiotherapy. In phantom pain, spinal cord thermolesion and stimulations of the peripheral nerves are effective, while neurodestruction is possible in stump pain..$^{32-34}$

\section{Post-traumatic Pain (PTP) and Post-operative Pain (PPP)}

PPP and PTP are chronic pathological pains which are associated with surgery or trauma and are present for more than normal tissue healing time, and their intensities, characters and localizations would not be explicated by the incident of other pathologies, for instance, disease recurrence, cancer or infection recurrence. ${ }^{35}$

Prophylactic measures prior to surgeries are recommended to reduce the risk of nerve damage; for instance, techniques for epidural anesthesia; using local anesthetics for surgical wounds; pregabalin or gabapentin use in perioperative phase

Despite the lack of evidence, it's recommended to give lidocaine intravenous infusions in all risk groups (young patients, long duration surgeries, poor treatment of severe post-operative pain).

Despite the lack of evidence, treatment of post-operative pain is:

- Causative management

- Five-percent lidocaine patches alone or in combination with systemic drugs

In case of PPP refractory to therapeutic drugs, it's possible to consider interventional methods to relieve the pain. ${ }^{1}$

\section{Neuropathic Pain - Cancer Patients}

Cancer patients' pain may have different causes and mechanisms (Table 1). Treatment choice depends on NP intensity where tramadol is administered in case of moderate intensity NP, while a strong opioid is given in cases of high intensity pain. Combination of the previous drugs with gabapentin and pregabalin helps in increasing effectiveness and reducing opioid doses. Venlofaxine and duloxetine are administered in treatment of neuropathy after chemotherapy. ${ }^{1,36}$

\section{Neuropathy - HIV Infection}

Neuropathy experienced by HIV patient is either due to the virus itself, or due to the anti-retroviral therapy. A combination of $8 \%$ capsaicin patch, lamotrigine and gabapentin are recommended. Nevertheless, $5 \%$ lidocaine patches, pregabalin and amitriptyline lack effectiveness in treating such pain. ${ }^{1,40}$

\section{Central Pain $(C P)$}

$\mathrm{CP}$ could be diagnosed in patients suffering from NP as a result of CNS injuries. It is crucial to indicate the etiology of such pain, e.g. Parkinson pain. The first-line agent in central pain therapy is pregabalin. However, in patients with depression or insomnia, the first-line treatment recommended are TCAs. ${ }^{1,3}$

\section{Nerve Injury and Neuropathic Pain}

The appearance of peripheral nerve injury on somatosensory processing and pain is directly related to age at which damage took place. ${ }^{41}$ The mechanical hypersensitivity of the patients does occur but only late in life. This delay hypersensitivity could be observed by cold stimulation and weight bearing tests. ${ }^{41}$ This pain could be attenuated by pre-treatment with, an inhibitor of pro-inflammatory polarised microglia, minocycline. $^{42,43}$ 
Raafat et al:: Neuropathic Pain and Potential Phytotherapies

Table 1: Causes and mechanisms of cancer patients pain, modified from [36].

\begin{tabular}{cccc} 
& Cancer Patient Cause of Pain & Mechanism/Cause & Reference \\
\hline I & Cancer Metastasis & Cancer infiltration to the PNS or CNS & {$[37]$} \\
II & Anticancer therapy & Nervous system intraoperative damage & {$[38]$} \\
III & Cancer related disease & Acute varicella zoster and post-herpetic neuralgia & {$[39]$} \\
IV & Disease(s) not related to cancer & Diabetic polyneuropathy & {$[1]$} \\
\hline
\end{tabular}

\section{Neuropathic pain in osteoarthritis}

The most common disorder of the muscle skeleton is osteoarthritis (OA), characterized by joint pain due to the central pain pathways and peripheral joint sensitization and the local joint inflammation done by nociceptive mechanism and changes in the bones. ${ }^{44}$ Thus, medicines acting centrally can relieve osteoarthritic pain, although osteoarthritic pain causes are not completely understood. ${ }^{45,46} \mathrm{OA}$ apparent mechanism of NP is due to increase in sodium channels, secondary hyperalgesia resulting from dorsal-horn sensitization of the NP modulation central point, and windup owing to the increase of calcium influx when NMDA receptors are activated. ${ }^{3,47,48}$

However, non-pharmaceutical treatment, as physiotherapy, hydrotherapy, and most important the use of electrical stimulation of nerves and cold or heat packs are essentially equal. Consequently, the centrally acting medications might be used together with anti-inflammatory and analgesic drugs for some patients in the future. Additional options like strontium ranelate/bisphosphonates for methotrexate are still under investigation. ${ }^{49}$

\section{METHODS}

\section{Search strategy}

The search in the primary literature has been performed by the leadresearcher (K. Raafat) between January and March 2016. A computerized based search has been accomplished in worldwide well established scientific databases mainly PUBMED and SCOPUS. Each database has been searched utilizing a search strategy developed of the combination of the keywords following: neuropathy or neuropathy pain or neuropathy diagnosis or neuropathy current therapies or neuropathy mechanisms or neuropathy extract or neuropathy phytotherapies or neuropathic pain therapy recommendations. The relevant research article citations have been investigated for the potential secondary and tertiary literature.

\section{Exclusion and inclusion criteria}

The following exclusion and inclusion criteria have been utilized; paper type: primary, secondary or tertiary literature, written in English, in peerreviewed journals or published books; study design: studies employing pre-clinical or clinical models research methods were included. Full text manuscripts of the included publications have been retrieved. In case of studies with identical results and methodologies, the earlier publication has been selected. The selection of phytotherapies was based upon their potentials in relieving NP in pre-clinical or clinical models. The authors of relevant studies have been contacted for any missing information, and studies have been excluded if no communication has been established.

\section{Quality assessment}

The included studies quality has been examined utilizing a standardized tool. The tool consists of eight components: (I) participants have been representative to the targeted population, (II) design of the study, (III) confounding factors control (IV) clinical trials blinding (V) data-collection reliability, (VI) the exclusion and inclusion criteria, (VII) integrity of intervention and (viii) analyses of the intervention. The studies have been included in the current study if all criteria have been fulfilled. On the other hand, the fourth criterion has been considered not feasible when assessing some pre-clinical studies. ${ }^{50}$

\section{Data synthesis}

In order to combine the data that have been included from the literature, a narrative synthesis method has been utilized. Electronic copies of manuscripts have been read by the lead-researcher to improve information extraction relevant to the inclusion and exclusion criteria and the manuscript characteristics, to examine their quality. At least two researchers have read each manuscript. The original research characteristics have been examined utilizing a pre-arranged scheme and the following information has been extracted: phytotherapy name and family, part used, aim of the study, most active constituents, highest dose utilized, analysis method, the route of administration, pre-clinical or clinical model, presence of positive control and its route of administration, results, neuropathic pain indication and limitations.

\section{MECHANISMS}

Literature has shown several mechanisms that cause the NP symptoms:

\section{Sensitization of nociceptors}

Exogenous or endogenous substances trigger the nociceptors. These substances involve inflammatory mediators (prostaglandins, bradykinin, and arachidonic acid different derivates), neurotransmitters (neurokinins, stimulatory-amino acids, noradrenalin, histamine and 5-HT), and growth factors. ${ }^{51-53}$

Upon the injury of peripheral nerve axon, degeneration takes place. Inflammatory mediators, growth factors, proinflammatory cytokines are released. As a result, hyperalgesia and allodynia occurs. ${ }^{54-56}$

Afferent neurons irregular ectopic excitability

Irregular excitability of $\mathrm{A} \delta$ and $\mathrm{C}$ fibers result in stinging and burning pain. Sodium channels apparently cause these symptoms. Moreover, voltage-gated ion channels, $\mathrm{Na}_{\mathrm{V}}(1.7,1.8$ and 1.9), are of particular role in pain perception. ${ }^{57-59}$

\section{Spinal dorsal horn pronociceptive facilitation}

Glutamate is a crucial stimulatory pain neurotransmitter caused by the CNS. The conduction of peripheral pain signals involves glutamate receptors. ${ }^{60-64}$ Calcitonin-gene related peptide and substance $\mathrm{P}$ are entangled in the disinhibition of the ionotropic-glutamate NMDA receptors. Moreover, metabotropic-glutamate receptors hinder the adenylyl-cyclase and decreases the conduction of pain signals..$^{62,65,66}$

\section{Spinal inhibitory network disinhibition of pain}

Thermal-grill illusion (TGI) has been provoked by the loss of inhibition through a non-noxious cold stimulus at the level of the spinal-cord. ${ }^{67,68}$ TGI was related to the loss of inhibition of central and peripheral toxic thermal responsive pathways..$^{62,69,70}$

\section{Sympathetically controlled pain}

Indirect and direct mechanisms are both involved in sympathetically controlled pain. Nociceptive input and efferent sympathetic signaling are coupled in neuropathic pain. The subcutaneous introduction of noradrenalin after continuous sympathetic block results in similar pain sensations. After sympathectomy, hyperalgesia and pain were the result of the sympathetic trunk stimulation. ${ }^{71,72}$ 
Also, findings show that in a few NP cases, sympathetic-activity might lead to pain stimulation. Indirect mechanisms are related to elevated sympathetically-stimulated vasomotor activity leading to impaired nutrition and oxygen supply with altered microcirculation. ${ }^{62,73}$

\section{Central reorganization phenomena}

Significant reorganization in imaging examinations of phantom-limb pain (PLP) patients was admitted. Pain intensity was strongly related to the quantity of cortical reorganization. This shows that the wider exposed body part that is not involved into extension field of the excised part, the higher the PLP. ${ }^{62}$

\section{CURRENT THERAPIES}

From a pharmacodynamics perspective, drugs treating neuropathic pain classes includes, anticonvulsants, antidepressants and antiarrhythmics. ${ }^{74}$ In spite of lack of information from controlled randomized trials, a number of drugs have shown clinically positive effects on specific NP symptoms (Table 2).

\section{SELECTED POTENTIAL PHYTOTHERAPIES}

Currently, phytotherapies have been utilized as an adjunctive therapy for amelioration of NP. ${ }^{76,77}$ Many studies have provided evidence underling the beneficial effects of phytotherapy in NP (Figure 1).

Nowadays, many pre-clinical and clinical trials have tested and utilized phytotherapy as an adjunct therapy for NP. ${ }^{76}$ Nevertheless, the efficacy of phytotherapy towards NP patients still been questioned. Therefore, we selected some frontline phytotherapies to review their efficacy towards NP (Table 3).

Table 2: Neuropathic pain lines of treatment, modified from ${ }^{75}$

\begin{tabular}{|c|c|c|}
\hline $\begin{array}{l}\text { Lines of } \\
\text { Treatment }\end{array}$ & Treatment & $\begin{array}{l}\text { Indication/ } \\
\text { Neuropathic pain } \\
\text { symptoms }\end{array}$ \\
\hline \multirow{11}{*}{ I } & Tricyclic antidepressants (TCAs): & \\
\hline & - Desipramine & BRP \\
\hline & - Amitriptyline & STP; BRP \\
\hline & - Imipramine & STP; BRP \\
\hline & $\begin{array}{l}\text { Serotonin and noradrenalin } \\
\text { reuptake inhibitors (SNRIs): }\end{array}$ & \\
\hline & - Venlafaxine & STP \\
\hline & Ca channel alpha 2 delta: & \\
\hline & - Pregabalin & ADA; HPA; STP; BRP \\
\hline & - Gabapentin & ADA; HPA; STP; BRP \\
\hline & Local anesthetic: & \\
\hline & - Topical 5\% lidocaine patches. & ADA; HPA \\
\hline \multirow{3}{*}{ II } & Opioids & \\
\hline & - Morphine & $\mathrm{ADA}$ \\
\hline & - Tramadol & $\mathrm{ADA}$ \\
\hline \multirow{5}{*}{ III } & Other anticonvulsants: & \\
\hline & - Carbamazepine & STP \\
\hline & - Lamotrigine & STP \\
\hline & Others: & \\
\hline & -Topical capsaicin & ADA; HPA \\
\hline
\end{tabular}

$\mathrm{ADA}=$ Allodynia; $\mathrm{HPA}=$ Hyperalgesia; $\mathrm{STP}=$ Shooting pain; $\mathrm{BRP}=$ Burning pain .

\section{Rheum ribes}

Rheum ribes ( $R$. ribes), the Syrian rhubarb, is traditionally utilized in Lebanese traditional medicine for DM management. $R$. ribes aqueous extract was investigated pre-clinically for antidiabetic, antioxidant and antidiabetic-neuropathy activities in experimental animals. ${ }^{78} R$. ribes highest dose $(50 \mathrm{mg} / \mathrm{kg})$ significantly elevated serum-CAT levels. Bio-guided fractionation proved that the most active compound present in $R$. ribes is rutin. Prophylactic treatment with $R$. ribes before alloxination, guarded against provoking diabetic neuropathy (DN) in experimental animals. In another diabetic group, after eight weeks of treatment, $R$. ribes significantly ameliorated hyperalgesia in experimental animals. These data pave the way for a future promising drug for the amelioration of $\mathrm{DN}$ and a novel prophylactic-treatment intended for $\mathrm{DN}$ vulnerable groups. $^{78,79}$

\section{Cannabinoids}

Chemotherapy is known to have a lot of serious side effects. One of these effects is severe NP, that makes quality of life impairment. Moreover, Chemotherapy evoked NP poorly responds to the conventional analgesics. ${ }^{94}$ Therefore, cannabinoid extract were tested for its effects on chemotherapy-induced NP treatment. ${ }^{95}, 96$ The drug used is nabiximol (delta-9 tetrahydrocannabinol with cannabidiol as spray) on sixteen patients, and a (0-10) numeric points rating-scale for NP intensity has been utilized as the main result measure. The primary outcome that five participants experienced reduction of pain by 2.6-3.4 points. Ten participants continued an extension phase and reported modest improvement. This pain reduction makes nabiximol an important candidate to do larger randomized trials ${ }^{94,95,97}$ Furthermore, a meta analysis was done on inhaled cannabis and its effect on NP in 405 patients in an individualized form. This study showed a $30 \%$ reduction in chronic pain improvement at the individual patient level..$^{80}$ Therefore, cannabinoids open doors for a future treatment for NP.

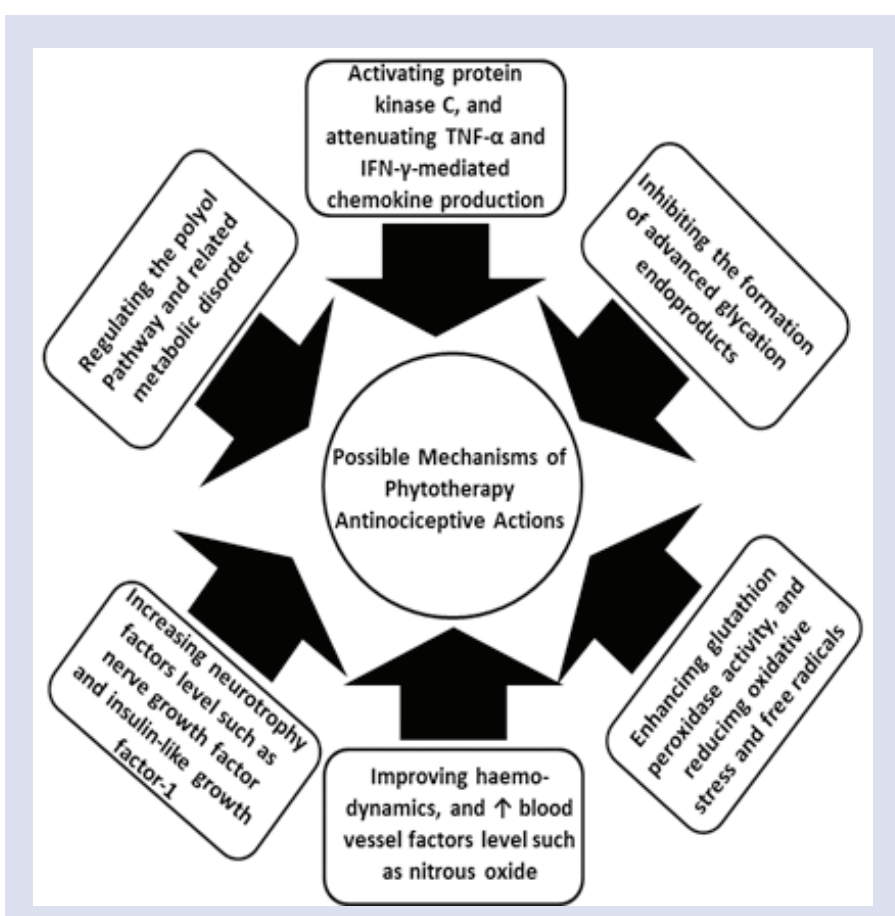

Figure 1: Possible mechanisms of phytotherapy antinociceptive actions modified from. ${ }^{76,77}$ 


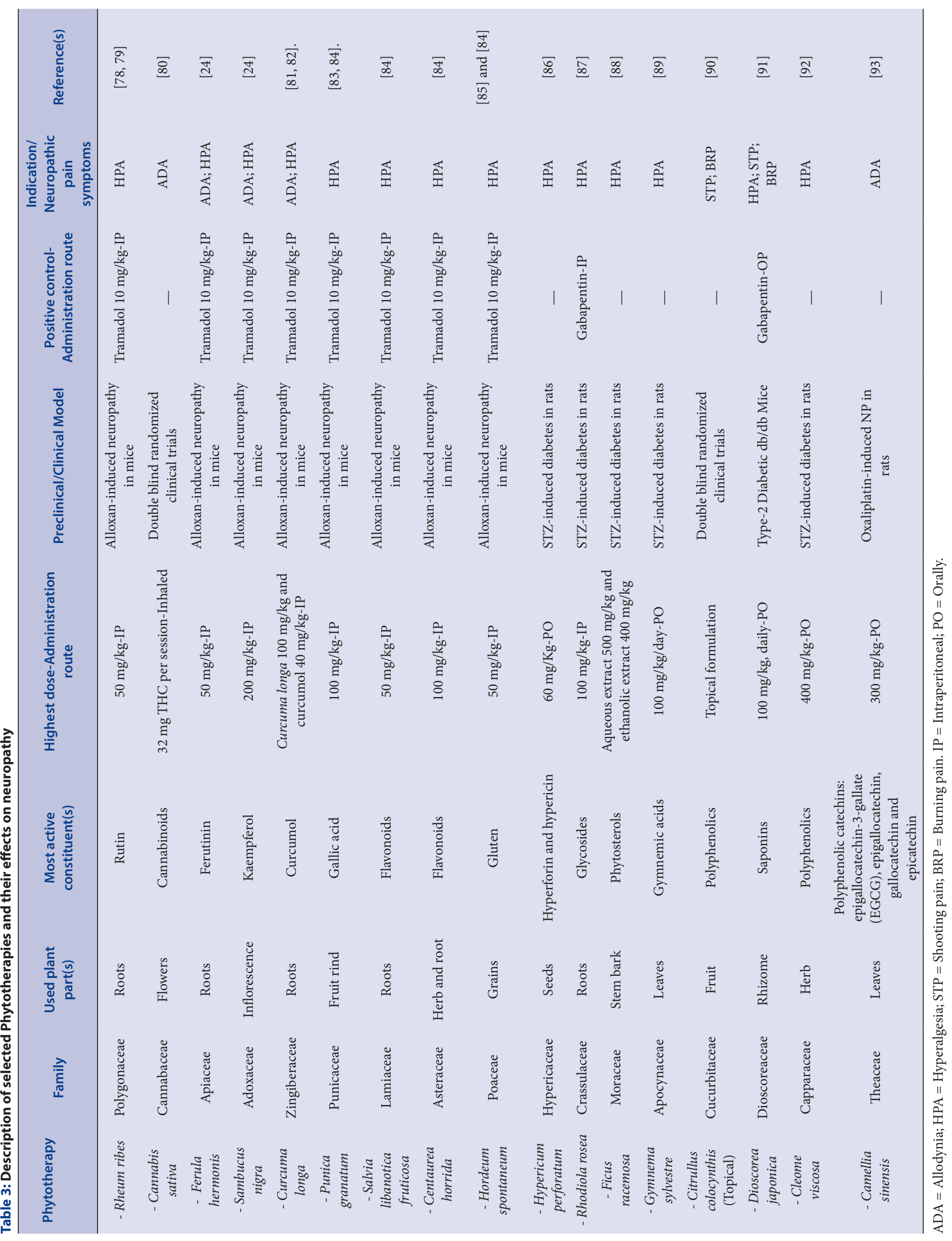




\section{Ferula hermonis}

Ferula hermonis (Ferula) or Zallouh roots, has been recognized for its sexual stimulant powers for many years. Ferula are known for the treatment of stomach disorders, skin infections, dysentery, fever and neurological disorders in the Asian folk medicine. ${ }^{98}$

In a recent study, the most active isolate utilizing bio-guided fractionation from Ferula was ferutinin. Compared to tramadol (TRA), the highest doses of tested Ferula $(50 \mathrm{mg} / \mathrm{kg}$ ) exerted remarkable effect towards ameliorating allodynia and thermal hyperalgesia. ${ }^{24}$

\section{Sambucus nigra}

Sambucus nigra (Elder) is another medicinal plant in which Elder is used in the management of diabetes neuropathic pain ${ }^{24}$ The inflorescences of Elder comprises valuable phenolic compounds, comprising flavonoids, phenolic acids, proanthocyanidins and catechins. ${ }^{99}$ Moreover, Elder demonstrates immune-stimulating, anticancer, ${ }^{100}$ antiallergic, antiviral, antibacterial, bronchodilatory, antitussive activity. ${ }^{101}$

Elder has shown significant effects towards amelioration of mechanical allodynia and hyperalgesia ${ }^{24}$ Elder were fractioned by RP-column chromatography and RP-HPLC, and showed that the most effective compound was kaempferol. Elder has shown high efficacy against tactile allodynia after a single-administration. However, for amelioration of hyperalgesia, needed repeated administration ${ }^{24}$

\section{Curcumol}

Turmeric (curcuma longa L.) contains curcumol, a guaiane-type sesquiterpenoid hemiketal. Curcumol could be used as antihepatic fibrosis, antiinflammatory, antitumor and anti microbial effects. ${ }^{102-105}$ Increasing oxidative stress will increase diabetic neuropathy because the tissues will not use the glucose present in blood. Supplementation of antioxidant compounds, like curcumol, ameliorates diabetic neuropathy. ${ }^{81,82}$

Pre-clinically treatment of mice with turmeric extract show high improvement in the management of diabetic neuropathy. Same goes to curcumol. Furthermore, they improve tactile allodynia. Rapid onset medication (turmeric) may be better for acute management. However, curcumol is efficient for long term management. ${ }^{81}$

In conclusion, based on the previous results, curcumol decreases oxidative stress and ameliorates the nerve functions in tactile allodynia.

\section{Punica granatum}

Due to its safety and availability, herbal extracts are being used in the regulation of diabetic neuropathy. ${ }^{83,84}$ One of these herbal extracts, is Punica granatum ethanolic extract (P. granatum) and its dried spray biopolymeric dispersion of either chitosan (F2) or casein (F1) has in vivo antioxidant and improves diabetic neuropathy complications. Pre-clinical studies for diabetic neuropathy, using $P$. granatum extract $(100 \mathrm{mg} / \mathrm{kg})$, F2, F1, and gallic acid $(6 \mathrm{mg} / \mathrm{kg})$, clearly enhanced thermal and tail flick latencies and ameliorated oxidative stress. ${ }^{106}$

\section{Salvia fruticosa}

Salvia or Salvia libanotica fruticosa (Lamiaceae) grows chiefly in Lebanon. ${ }^{107}$ Pre-clinical studies suggests that Salvia improves peripheral nerve function in the diabetic mice. ${ }^{84,} 108$ These findings give clinicians promising drug-leads for the management of diabetic neuropathy symptoms.

\section{Centaurea horrida}

Centaurea horrida is another antioxidant rich plant. Extracts from leaves, flowers ${ }^{109}$ and roots. ${ }^{84}$ were reported to depress blood sugar levels and ameliorate thermal hyperalgesia in mice models of diabetic neuropathy.

\section{Hordeum spontaneum}

Hordeum spontaneum K. (Poaceae), known as wild barley is known for its hypoglycemic potentials. ${ }^{85}$ Its high levels of beta-glucan which causes alleviation of insulin levels and decrease of oxidative stress might the underlying causes of wild barley diabetic neuropathy ameliorative effects. $^{84}$

\section{Hypericum perforatum}

Acute administration of Hypericum perforatum, St. John's Wort seed extract (SJW), containing hyperforin and hypericin, ameliorated mechanical hyperalgesia for a prolonged time. The antinociceptive efficacy of SJW was comparable to that of current therapies (carbamazepine and L-acetyllevocarnitine). ${ }^{86}$ These results suggest that SJW might become a novel therapeutic perspective for painful diabetic neuropathy.

\section{Rhodiola rosea}

Golden root or Rhodiola rosea ethanol extract (R. rosea) (Crassulaceae) preclinical studies has shown a significant activity towards thermal and chemical antinociceptive activities, suggesting that $R$. rosea had antidiabetic hyperalgesia potentials. ${ }^{87}$

\section{Ficus racemosa}

Preclinical study showed Ficus racemosa had a significant antioxidant potential and marked improvement in hyperalgesia utilizing locomotion rise, tail flick and fall latency periods ${ }^{88}$ Further clinical studies are required to support these findings.

\section{Gymnema sylvestre}

Gymnema sylvestre treated diabetic animals had shown neuroprotective effects associated with antioxidant and antinflammatory activities. ${ }^{89}$ It has proved to be one of most promising diabetic neuropathy future preventive therapies.

\section{Citrullus colocynthis}

Bitter apple or Citrullus colocynthis (C. colocynthis) is one of the fruits that has shown significant potentials against diabetic NP. In a doubleblind placebo-controlled clinical randomized trial, C. colocynthis topical formulation had a significant antinociceptive effects against painful diabetic neuropathy. ${ }^{90}$ More studies are required to establish C. colocynthis potentials on nerve function and overall quality of life.

\section{Dioscorea japonica}

Standardized Dioscorea japonica extract has shown to ameliorate, transgenic diabetic animals, thermal hyperalgesia and peripheral-nerve damage. ${ }^{91}$ These results might pave the way for preparation of a potential botanical therapy for diabetic neuropathy.

\section{Cleome viscosa}

In vivo subchronic treatment with Cleome viscosa ethanolic extract significantly attenuated nociceptive thresholds. It had also significant in vivo antioxidant activities. ${ }^{92}$ Additional work is suggested to know its antinociceptive mechanism of action.

\section{Camellia sinensis}

Green tea has been found to contain four major polyphenolic catechins: epigallocatechin-3-gallate (EGCG), epigallocatechin, gallocatechin and epicatechin. ${ }^{110}$ Because of the antioxidant activity of its polyphenol catechins, Camellia sinensis has been found to be useful adjuvant to alleviate oxaliplatin-induced allodynic sensory symptoms in a rat model of neuropathic pain..$^{93}$ More clinical trials are required to strengthen these findings.

Finally, these data has shown that natural therapies have indeed significant potentials to be the future hope for NP patients. However, the management of neuropathic pain remains to be an ongoing battle in the 
purpose of finding the optimum treatment with the best benefit on the patients' life quality.

\section{DISCUSSION}

In spite of the existence of guidelines and a number of effective drugs for the treatment of NP, there are still several cases that remain untreated or undertreated. This concern has highlighted the need of several research studies in the scientific field. One of these studies is the management of NP through phytotherapies.

Oxidative stress has been involved in the reactive oxygen species (ROS) release, responsible for many neurodegenerative disorders including NP. ${ }^{111}$ The cellular defensive mechanism opposing ROS-mediated cellular injury has been ameliorated by the natural antioxidant enzymes. Phytotherapies such as Rheum ribes, Cannabis sativa, Ferula hermonis, Sambucus nigra, Curcuma longa, Punica granatum, Salvia libanotica fruticosa, Centaurea horrida, Hordeum spontaneum, Hypericum perforatum, Rhodiola rosea, Ficus racemosa, Gymnema sylvestre, Citrullus colocynthis, Dioscorea japonica, Camellia sinensis and Cleome viscosa had shown the ability to ameliorate NP by various mechanisms of actions. Most of these medicinal plants have shown to comprise bioactive constituents possessing antioxidant potentials.

$R$. ribes has shown to be one of the most potent phytotherapies. Bio-guided fractionation has proved that rutin, a key constituent of $R$. ribes, significantly decreased hyperalgesia (Table 3 ), and was suggested that this effect is due to elevation of natural antioxidant effect, catalase. ${ }^{78}$ Nevertheless, there is no full clinical evidence for its use. On the other hand, a number of conventional drugs have shown clinically positive effects on specific NP symptoms (Table 2).

C. sativa has also shown potent neuroprotective effects. It has been investigated in terms of amelioration of NP in the literature review (Table 3). Double blind randomized clinical trials have shown that inhaled THC, a key constituent of $C$. sativa, significantly decreased allodynia, and was suggested that this effect is due to THC specific suppression of central and peripheral sensitization..$^{80,96}$

The third most potent herb was F. hermonis. Interestingly, there has been no detailed clinical studies investigating $F$. hermonis in the amelioration of NP reported in the English language. In vivo pharmacological investigation had shown that ferutinin, a constituent of F. hermonis, significantly decreased hyperalgesia and allodynia, and was suggested that this effect is due to elevation of natural antioxidant effect, catalase ${ }^{24}$ (Table 3). S. nigra has shown potent antihyperalgesic and antiallodynic activity. Although no detailed clinical trial has been reported, the bio-guided fractionation has shown that kampferol was the most active constituent in the inflorescence of $S$. nigra. Literature review has shown that kampferol antioxidant potentials might be responsible for $S$. nigra neuroprotective effects. ${ }^{24}$

The research performed on C. longa has indicated efficacy in NP amelioration. Pharmacological studies on mice have indicated that the antioxidant potentials of curcumol, C. longa key constituent, alleviated allodynia and hyperalgesia. ${ }^{81}$ (Table 3 ). Detailed clinical trials are required to fully understand curcumol mechanism of action.

Research on the utilization of $P$. granatum has shown to be a promising phytotherapeutic agent for amelioration of NP. The antioxidant potentials of gallic acid, $P$. granatum key constituent, has shown effective antihyperalgesic activity. ${ }^{106} \mathrm{~A}$ much extended and larger clinical trials would be recommended to fully investigate $P$. granatum neuroprotective activities. The potential significant role of ethnopharmacology in the evolution of herbal treatment for NP has been shown by research on three herbs. An in vivo investigation was performed by three traditional herbs, namely S. fruticosa, C. horrida and H. spontaneum. Among these herbs, C. horrida has shown to possess the most potent antihyperalgesic activity. C. horrida has potentially elevated catalase levels suggesting that its antioxidant potential might be responsible for its neuroprotective effects. ${ }^{84}$

H. perforatum has shown potent antihyperalgesic activity against NP in a diabetic rat model. Research so far has shown that hyperforin and hypericin, $H$. perforatum key constituents, might be responsible for its neuroprotective activity. ${ }^{86}$ (Table 3 ). These results have been only demonstrated in rat model, however, the results appear as a promising future treatment.

The traditional use of $R$. rosea has led the scientists to discover its antihyperalgesic potentials. Preclinical studies has shown that $R$. rosea inhibited COX-1 and -2 and phospholipase A2, suggesting that this mechanism is responsible for antinflammatory and antinociceptive activities ${ }^{87}$ (Table 3). These finding reflect positive outcomes for further investigation.

The protective role of $F$. racemosa against neurodegenerative NP has been demonstrated in a rat model. The antioxidant potentials of $F$. racemosa monitored in the significant elevation in catalase, superoxide dismutase, and amelioration of nitrite level, C-reactive protein and lipid peroxidation levels, which might be responsible for its potential antihyperalgesic activity. ${ }^{88}$

Gymnemic acids, the active constituents in G. sylvestre, has proved to have antihyperalgesic activity in a preclinical model. The association of gymnemic acids antioxidant and antinflammatory activities might be responsible for its neuroprotective effects. ${ }^{89}$ Further clinical studies are indicated for fully understanding gymnemic acids mechanism of action against NP.

Within the inclusion criteria of this study, C. colocynthis has been appropriately researched. In a double-blind placebo-controlled clinical randomized trial, C. colocynthis topical formulation had a significant antinociceptive effects against painful diabetic neuropathy. ${ }^{90}$ Further studies are required to establish C. colocynthis potentials on overall quality of life.

D. japonica alleviated transgenic diabetic animals, thermal hyperalgesia and peripheral-nerve damage. Induction of nerve growth factor might be responsible for $D$. japonica extract ameliorative effects towards hyperalgesia, shooting and burning pains. Latest research has indicated that D. japonica standardized extract were being under investigation in a randomized clinical study. ${ }^{91}$ This study results might pave the way for validation of $D$. japonica use against NP.

The significant antioxidant activity was responsible of the choice of C. viscosa as a potential candidate in amelioration of NP. Preclinical studies have shown that $C$. viscosa possessed significant antihyperalgesic properties. Moreover, the amelioration of lipid peroxidation and oxidative stress in treated NP models, has suggested their association in C. viscosa mechanism of action. ${ }^{92}$

In addition, even though green tea has shown temporary effect on neurotoxicity, it might be a useful future adjuvant therapy to ameliorate allodynic sensory symptoms in the primary stages of preclinical neurotoxicity with a good safety history. ${ }^{93}$ Nevertheless, more in-depth clinical studies are required to validate green tea utilization in NP management.

Generally, herbal therapy appeared to be well tolerated by preclinical/ clinical models with NP. Potential side effects of these natural plants had not shown as an emerging issue. However, these phytotherapies need more clinical evidence to demonstrate any underlying side effects, and to verify their promising neuroprotective properties.

Moreover, the study results have indicated the potential advantages of phytotherapy as a NP therapeutic option, utilized both alone or in combination with conventional treatment. This study also suggested that 
further investigation into the utilization of phytotherapy in the treatment of NP was both needed and justified.

\section{RECOMMENDATIONS IN MANAGEMENT OF NEUROPATHIC PAIN}

I. Pain management should be headed by knowing the cause of NP and initiating a suitable treatment.

II. Relevant comorbidities should be identified as they may require analgesic dosage modification or constitute contraindications for treatment.

III. Patient education should be implemented (like; treatment and diagnosis).

IV. Specific NP syndromes according to lines of treatment (Table 2 and 3) should be managed with regards to the recent recommendations.

V. First-line treatment should be continued if it is effective (at least $50 \%$ pain reduction), in an adequate time.

VI. Lack of effectiveness of first-line drugs, used at appropriate doses either alone or in combination, designates the need for trying treatment with second-line and third-line drugs.

VII. Pharmacologic management should be accompanied with nonpharmacologic interventions.

VIII. Treatment efficiency should be measured with suitable frequency.

IX. Physicians consider spinal cord stimulation SCS, is a factor to block stimulus transmission, or intrathecal drug delivery systems when other pharmacological treatments fail.

$\mathrm{X}$. Alternative treatments include phytotherapies are needed to decrease the dose and possible side-effects of the first line treatments.

XI. Phytotherapies need more clinical trials in order to fully understand their mechanism of action, to demonstrate any underlying side effects, and to validate their future use.

\section{CONCLUSION}

In spite of the presence of guidelines and a number of effective drugs for the treatment of NP, there are still several cases that remain untreated or undertreated. This issue has raised the concern of several research studies in the scientific field. One of these measures is the management of NP through phytotherapy. Therefore, this review discusses neuropathic pain and provides multiple recommendations on how to diagnose and manage this syndrome with a special emphasis on phytotherapy potentials. Phytotherapies, although not yet well studied, offering relatively low-risk options in neuropathic pain patients and having an increasing evidence to be the future of NP management.

\section{ACKNOWLEDGMENT}

Authors are very grateful to Prof. A. EL-Lakany and Prof. M. Abou-Ela for supporting Faculty of Pharmacy, Beirut Arab University junior research team. Thanks are also due to Mrs. G. Onsy for proof-reading the manuscript.

\section{CONFLICTS OF INTEREST}

There are no conflicts of interest.

\section{ABBREVIATION USED}

NP; neuropathic pain; CNS; central nervous system; TN; Trigeminal Neuralgia; CP; Central Pain; TCAs; Tricyclic antidepressants; OA; Osteoarthritis; TGI; Thermal-grill illusion; PLP; phantom-limb pain; ROS; reactive oxygen species; COX; Cyclooxygenase.

\section{REFERENCES}

1. Szczudlik A, et al. Diagnosis and management of neuropathic pain: review of literature and recommendations of the Polish Association for the Study of Pain and the Polish Neurological Society-Part Two. Neurol Neurochir Pol. 2014;48(6): 423-35.

2. Stepien A, Domzał TM. Nowe wytyczne dotyczace leczenia bólu neuropatycznego. Neurologia i Neurochirurgia Polska. 2010;44(5):437-42

3. Treede RD. et al. Neuropathic pain: redefinition and a grading system for clinical and research purposes. Neurology. 2008;70(18):1630-5.

4. Torrance $N$, et al. The epidemiology of chronic pain of predominantly neuropathic origin. Results from a general population survey. J Pain. 2006;7(4):281-9.

5. Bouhassira $D$, et al. Prevalence of chronic pain with neuropathic characteristics in the general population. Pain. 2008;136(3):380-7.

6. Attal N. et al. The specific disease burden of neuropathic pain: results of a French nationwide survey. Pain. 2011;152(12):2836-43

7. Langley PC. The prevalence, correlates and treatment of pain in the European Union. Curr Med Res Opin. 2011;27(2):463-80.

8. Smith $\mathrm{BH}$, Torrance N. Epidemiology of neuropathic pain and its impact on quality of life. Curr Pain Headache Rep. 2012;16(3):191-8.

9. Torrance $\mathrm{N}$, et al. Neuropathic pain in the community: more under-treated than refractory?. Pain. 2013;154(5):690-9.

10. Basbaum Al, et al. Cellular and molecular mechanisms of pain. Cell. 2009;139(2):267-84

11. Dworkin $\mathrm{RH}$, Portenoy RK. Pain and its persistence in herpes zoster. Pain. 1996;67(2-3):241-51

12. Thyregod HG, et al. Natural history of pain following herpes zoster. Pain. 2007;128(1-2):148-56

13. Jung $B F$, et al. Risk factors for postherpetic neuralgia in patients with herpes zoster. Neurology. 2004;62(9):1545-51.

14. Harden RN, et al, Proposed new diagnostic criteria for complex regional pain syndrome. Pain Med. 2007;8(4):326-31.

15. Zollinger $P E$, et al. Can vitamin $C$ prevent complex regional pain syndrome in patients with wrist fractures? A randomized, controlled, multicenter dose-response study. J Bone Joint Surg Am. 2007;89(7):1424-31.

16. Lee $\mathrm{BH}$, et al. Physical therapy and cognitive-behavioral treatment for complex regional pain syndromes. J Pediatr. 2002;141(1):135-40.

17. Moseley GL. Is successful rehabilitation of complex regional pain syndrome due to sustained attention to the affected limb? A randomised clinical trial. Pain. 2005; 114(1-2):54-61.

18. Oerlemans HM, et al. Evaluation of three methods to rate impairment in patients with complex regional pain syndrome I of one upper extremity. Clin Rehabil. 2000;14(3):331-9.

19. Partanen J, et al. Natural history of peripheral neuropathy in patients with noninsulin-dependent diabetes mellitus. N Engl J Med. 1995;333(2):89-94.

20. Zychowska $M$, et al. Mechanisms and pharmacology of diabetic neuropathy experimental and clinical studies. Pharmacol Rep. 2013;65(6):1601-10.

21. Boulton AJM, et al. Diabetic Neuropathies: A statement by the American Diabetes Association. Diabetes Care. 2005;28(4):956-62.

22. Bril $\mathrm{V}$, et al. Evidence-based guideline: Treatment of painful diabetic neuropathy: report of the American Academy of Neurology, the American Association of Neuromuscular and Electrodiagnostic Medicine, and the American Academy of Physical Medicine and Rehabilitation. Neurology. 2011;76(20):1758-65.

23. Tesfaye $S$, et al. Painful diabetic peripheral neuropathy: consensus recommendations on diagnosis, assessment and management. Diabetes/Metabolism Research and Reviews. 2011;27(7):629-38.

24. Raafat K, El-Lakany A. Acute and subchronic in-vivo effects of Ferula hermonis L. and Sambucus nigra L. and their potential active isolates in a diabetic mouse model of neuropathic pain. BMC Complement Altern Med. 2015;15:257

25. Koopman JS, et al. Incidence of facial pain in the general population. Pain. 2009;147(1-3):122-7.

26. Zakrzewska JM. Diagnosis and differential diagnosis of trigeminal neuralgia. Clin J Pain. 2002;18(1):14-21.

27. Childs EA, et al. Plasma viral load and CD lymphocytes predict HIV-associated dementia and sensory neuropathy. Neurology. 1999;52(3):607-13.

28. Gronseth $\mathrm{G}$, et al. Practice parameter: the diagnostic evaluation and treatment of trigeminal neuralgia (an evidence-based review): report of the Quality Standards Subcommittee of the American Academy of Neurology and the European Federation of Neurological Societies. Neurology. 2008;71(15):1183-90.

29. Cruccu G, Truini A, Refractory trigeminal neuralgia. Non-surgical treatment options. CNS Drugs. 2013;27(2):91-6.

30. Koopman JSHA, et al. A nationwide study of three invasive treatments for trigeminal neuralgia. Pain. 2011;152(3):507-13.

31. Rauck RL, et al. Treatment of post-amputation pain with peripheral nerve stimulation. Neuromodulation. 2014. 17(2): p. 188-97.

32. Rosenstock J, et al. Pregabalin for the treatment of painful diabetic periphera neuropathy: a double-blind, placebo-controlled trial. Pain. 2004;110(3):628-38. 
33. Wilder-Smith CH, Hill LT, Laurent S. Postamputation pain and sensory changes in treatment-naive patients: characteristics and responses to treatment with tramadol, amitriptyline, and placebo. Anesthesiology. 2005;103(3):619-28.

34. Knotkova $\mathrm{H}$, et al. Current and future options for the management of phantomlimb pain. J Pain Res. 2012;5:39-49.

35. Perkins F, Ballantyne J. Postsurgical Pain Syndromes, in Evidence-Based Chronic Pain Management. 2010, John Wiley \& Sons, Ltd. 194-203.

36. Fallon MT. Neuropathic pain in cancer. Br J Anaesth. 2013;111(1):105-11.

37. Hamamoto $Y$, et al. Vertebral metastases with high risk of symptomatic malignant spinal cord compression. Jpn J Clin Oncol. 2009;39(7):431-4.

38. Vadivelu $\mathrm{N}$, et al. Pain after mastectomy and breast reconstruction. Am Surg. 2008;74(4):285-96

39. Dworkin $\mathrm{RH}$, et al. Recommendations for the management of herpes zoster. Clin Infect Dis. 2007;44 (Suppl 1):S1-26.

40. Verma A. Epidemiology and clinical features of HIV-1 associated neuropathies. J Peripher Nerv Syst. 2001;6(1):8-13

41. Fitzgerald $M$, McKelvey $R$, Nerve injury and neuropathic pain - A question of age. Exp Neurol. 2016;275 Pt 2:296-302.

42. Hathway GJ, et al. Brief, low frequency stimulation of rat peripheral C-fibres evokes prolonged microglial-induced central sensitization in adults but not in neonates. Pain. 2009;144(1-2):110-8.

43. Kobayashi K. et al. Minocycline selectively inhibits $M_{1}$ polarization of microglia. Cell Death Dis. 2013:4:e525.

44. Sharma L, Kapoor D, Issa S, Epidemiology of osteoarthritis: an update. Curr Opin Rheumatol. 2006;18(2):147-56.

45. Zhang J, et al. Risk factors for and prevalence of knee osteoarthritis in the rural areas of Shanxi Province, North China: a COPCORD study. Rheumatol Int. 2013;33(11):2783-8.

46. Nho SJ, et al. The burden of hip osteoarthritis in the United States: epidemiologic and economic considerations. J Am Acad Orthop Surg. 2013;21 Suppl 1:S1-6.

47. Sommer C. Painful neuropathies. Curr Opin Neurol. 2003;16(5):623-8.

48. Moore KA, et al. Partial peripheral nerve injury promotes a selective loss of GABAergic inhibition in the superficial dorsal horn of the spinal cord. J Neurosci. 2002;22(15):6724-31.

49. Dimitroulas T, et al. Neuropathic pain in osteoarthritis: a review of pathophysiological mechanisms and implications for treatment. Semin Arthritis Rheum. 2014;44(2):145-54.

50. MacRae S, et al. Diabetes in people with intellectual disabilities: A systematic review of the literature. Res Dev Disabil. 2015;47:352-74.

51. Julius D, Basbaum Al. Molecular mechanisms of nociception. Nature. 2001;413(6852):203-10.

52. Schmidt $R$, et al. Novel classes of responsive and unresponsive $C$ nociceptors in human skin. J Neurosci. 1995;15(1 Pt 1):333-41.

53. Ueda H. Peripheral mechanisms of neuropathic pain-involvement of lysophosphatidic acid receptor-mediated demyelination. Mol Pain. 2008;4:11.

54. Inoue $M$, et al. Initiation of neuropathic pain requires lysophosphatidic acid receptor signaling. Nat Med. 2004;10(7):712-8.

55. Moalem G, Tracey DJ. Immune and inflammatory mechanisms in neuropathic pain. Brain Res Rev. 2006;51(2):240-64.

56. Scholz J, Woolf CJ. The neuropathic pain triad: neurons, immune cells and glia. Nat Neurosci. 2007;10(11):1361-8.

57. Amir R, Michaelis M, Devor M. Membrane potential oscillations in dorsal root ganglion neurons: role in normal electrogenesis and neuropathic pain. J Neurosci. 1999;19(19):8589-96.

58. Tal M, Wall PD, Devor M. Myelinated afferent fiber types that become spontaneously active and mechanosensitive following nerve transection in the rat. Brain Res. 1999;824(2):218-23.

59. Cummins TR, Sheets PL, Waxman SG. The roles of sodium channels in nociception: Implications for mechanisms of pain. Pain. 2007;131(3):243-57.

60. D'Mello R, Dickenson AH. Spinal cord mechanisms of pain. Br J Anaesth. 2008;101(1):8-16.

61. Khasabov SG, et al. Spinal neurons that possess the substance $P$ receptor are required for the development of central sensitization. J Neurosci. 2002;22(20):9086-98.

62. Nickel FT, et al. Mechanisms of neuropathic pain. Eur Neuropsychopharmacol. 2012;22(2):81-91.

63. Suzuki R, Hunt SP, Dickenson AH. The coding of noxious mechanical and thermal stimuli of deep dorsal horn neurones is attenuated in NK1 knockout mice. Neuropharmacology. 2003;45(8):1093-100.

64. Pan $\mathrm{HL}$, et al. Modulation of pain transmission by G-protein-coupled receptors. Pharmacol Ther. 2008;117(1):141-61.

65. Fundytus $\mathrm{ME}$, et al. In vivo antinociceptive activity of anti-rat mGluR1 and mGluR5 antibodies in rats. Neuroreport. 1998;9(4):731-5.

66. Wu WL, et al. Discovery of orally efficacious tetracyclic metabotropic glutamate receptor 1 (mGluR1) antagonists for the treatment of chronic pain. J Med Chem. 2007;50(23):5550-3.
67. Craig AD, Bushnell MC. The thermal grill illusion: unmasking the burn of cold pain. Science. 1994;265(5169):252-5.

68. Susser E, Sprecher E, Yarnitsky D. Paradoxical heat sensation in healthy subjects: peripherally conducted by A delta or C fibres?. Brain. 1999;122 (Pt 2):239-46.

69. Knabl J, et al. Reversal of pathological pain through specific spinal GABAA receptor subtypes. Nature. 2008;451(7176):330-4

70. Zeilhofer HU, Ralvenius WT, Acuna MA. Restoring the spinal pain gate: GABA(A) receptors as targets for novel analgesics. Adv Pharmacol. 2015;73:71-96.

71. Sato J, Perl ER. Adrenergic excitation of cutaneous pain receptors induced by peripheral nerve injury. Science. 1991;251(5001):1608-10.

72. Torebjork E, et al. Noradrenaline-evoked pain in neuralgia. Pain. 1995;63(1):1120.

73. Cepeda MS, Carr DB, Lau J. Local anesthetic sympathetic blockade for complex regional pain syndrome. Cochrane Database Syst Rev. 2005(4):CD004598.

74. Carter GT, Galer BS. Advances in the management of neuropathic pain. Phys Med Rehabil Clin N Am. 2001;12(2):447-59.

75. Scholten PM, Harden RN. Assessing and Treating Patients With Neuropathic Pain. PMR. 2015;7(11 Suppl):S257-69.

76. $\mathrm{Xu} \mathrm{HB}$, et al. Chinese herbal medicine in treatment of diabetic peripheral neuropathy: a systematic review and meta-analysis. J Ethnopharmacol. 2012;143(2):701-8

77. Wu QL, Liang XC. [Survey of current experimental studies of effects of traditional Chinese compound recipe on diabetic peripheral neuropathy]. Zhongguo Zhong Yao Za Zhi. 2007;32(9):775-8.

78. Raafat K, Aboul-Ela M, El-Lakany A. Alloxan-induced diabetic thermal hyperalgesia, prophylaxis and phytotherapeutic effects of Rheum ribes $L$. in mouse model. Arch Pharm Res. 2014.

79. Vincent AM, et al. The antioxidant response as a drug target in diabetic neuropathy. Curr Drug Targets. 2008;9(1):94-100.

80. Andreae $\mathrm{MH}$, et al. Inhaled Cannabis for Chronic Neuropathic Pain: A Metaanalysis of Individual Patient Data. J Pain. 2015;16(12):1221-32.

81. Raafat KM, Omar AG. Phytotherapeutic activity of curcumol: Isolation, GC-MS identification, and assessing potentials against acute and subchronic hyperglycemia, tactile allodynia, and hyperalgesia. Pharm Biol. 2015:1-11.

82. Rahimi $R$, et al. A review on the role of antioxidants in the management of diabetes and its complications. Biomed Pharmacother. 2005;59(7):365-73.

83. Bakirel T, et al. In vivo assessment of antidiabetic and antioxidant activities of rosemary (Rosmarinus officinalis) in alloxan-diabetic rabbits. J Ethnopharmacol. 2008;116(1):64-73.

84. Raafat K, et al. Endogenous Lebanese Plants Treating Diabetes and Related Complications. Nat Prod Chem Res. 2013;1(3):112-20.

85. Volis S, et al. Phenotypic and allozyme variation in Mediterranean and desert populations of wild barley, Hordeum spontaneum Koch. Evolution. 2002;56(7):1403-15

86. Galeotti N, et al. St. John's Wort seed and feverfew flower extracts relieve painful diabetic neuropathy in a rat model of diabetes. Fitoterapia. 2014;92:23-33.

87. Deciga-Campos M, et al. Antihyperalgesic Activity of Rhodiola rosea in a Diabetic Rat Model. Drug Dev Res. 2016;77(1):29-36.

88. Solanki ND, Bhavsar SK. An evaluation of the protective role of Ficus racemosa Linn. in streptozotocin-induced diabetic neuropathy with neurodegeneration. Indian J Pharmacol. 2015;47(6):610-5.

89. Fatani AJ, et al. Neuroprotective effects of Gymnema Sylvestrae on streptozotocin-induced diabetic neuropathy in rats. Exp Ther Med. 2015;9(5):1670-8.

90. Heydari M, et al, Topical Citrullus colocynthis (bitter apple) extract oil in painful diabetic neuropathy: A double-blind randomized placebo-controlled clinical trial. J Diabetes. 2015

91. Moon E, et al. Dioscorea Extract (DA-9801) Modulates Markers of Peripheral Neuropathy in Type 2 Diabetic db/db Mice. Biomol Ther (Seoul). 2014;22(5): 44552.

92. Rao BS, et al. Effects of Cleome viscosa on hyperalgesia, oxidative stress and lipid profile in STZ induced diabetic neuropathy in Wistar rats. Pak J Pharm Sci. 2014;27(5):1137-45.

93. Lee JS, et al. Effect of green tea extracts on oxaliplatin-induced peripheral neuropathy in rats. BMC Complement Altern Med. 2012;12:124

94. Cata JP, et al. Clinical and experimental findings in humans and animals with chemotherapy-induced peripheral neuropathy. Minerva Anestesiol. 2006;72(3):151-69.

95. Polomano RC, Bennett GJ. Chemotherapy-evoked painful peripheral neuropathy. Pain Med. 2001;2(1):8-14

96. Lynch ME, Cesar-Rittenberg P, Hohmann AG. A double-blind, placebo-controlled, crossover pilot trial with extension using an oral mucosal cannabinoid extract for treatment of chemotherapy-induced neuropathic pain. J Pain Symptom Manage. 2014;47(1):166-73.

97. Dougherty PM, et al. Taxol-induced sensory disturbance is characterized by preferential impairment of myelinated fiber function in cancer patients. Pain. 2004;109(1-2):132-42. 
98. Gamal-Eldeen AM, Hegazy ME. A crystal lapiferin derived from Ferula vesceritensis induces apoptosis pathway in MCF-7 breast cancer cells. Nat Prod Res. 2010;24(3):246-57.

99. Veberic R, et al. European elderberry (Sambucus nigra L.) rich in sugars, organic acids, anthocyanins and selected polyphenols. Food Chem. 2009;114:511-5.

100. Halama $A$, et al. Polymer-based non-viral gene delivery as a concept for the treatment of cancer. Pharmacol Rep. 2009;61(6):993-9.

101. Sutovska M, et al, Antitussive and bronchodilatory effects of Lythrum salicaria polysaccharide-polyphenolic conjugate. Int J Biol Macromol. 2012;51(5):794-9.

102. Liu Y, et al. Functional food quality of Curcuma caesia, Curcuma zedoaria and Curcuma aeruginosa endemic to Northeastern India. Plant Foods Hum Nutr. 2013;68(1):72-7.

103. Kuroda $M$, et al. Hypoglycemic effects of turmeric (Curcuma longa L. rhizomes) on genetically diabetic KK-Ay mice. Biol Pharm Bull. 2005;28(5):937 9

104. Jiang $\mathrm{J}$, et al. [Effect of FZHY recipe on elastase expression in liver fibrosis in rats]. Zhonghua Gan Zang Bing Za Zhi. 2005;13(4):307-8.

105. Lou Y, et al. Isolation and identification of phase 1 metabolites of curcumol in rats. Drug Metab Dispos. 2010;38(11):2014-22

106. Raafat K, Samy W. Amelioration of Diabetes and Painful Diabetic Neuropathy by Punica granatum L. Extract and Its Spray Dried Biopolymeric Dispersions. Evid Based Complement Alternat Med. 2014;2014:180495.

107. Gali-Muhtasib H, Hilan C, Khater C. Traditional uses of Salvia libanotica (East Mediterranean sage) and the effects of its essential oils. J Ethnopharmacol 2000;71(3):513-20.

108. Perfumi M, Arnold N, Tacconi R. Hypoglycemic activity of Salvia fruticosa Mill. from Cyprus. J Ethnopharmacol. 1991;34(2-3):135-40.

109. Flamini G, et al. A new flavonoid glycoside from Centaurea horrida. J Nat Prod. 2000;63(5):662-3

110. Nanjo $F$, et al. Radical scavenging activity of tea catechins and their related compounds. Biosci Biotechnol Biochem. 1999;63(9):1621-3.

111. Valko $\mathrm{M}$, et al. Free radicals and antioxidants in normal physiological functions and human disease. Int J Biochem Cell Biol. 2007:39(1):44-84.

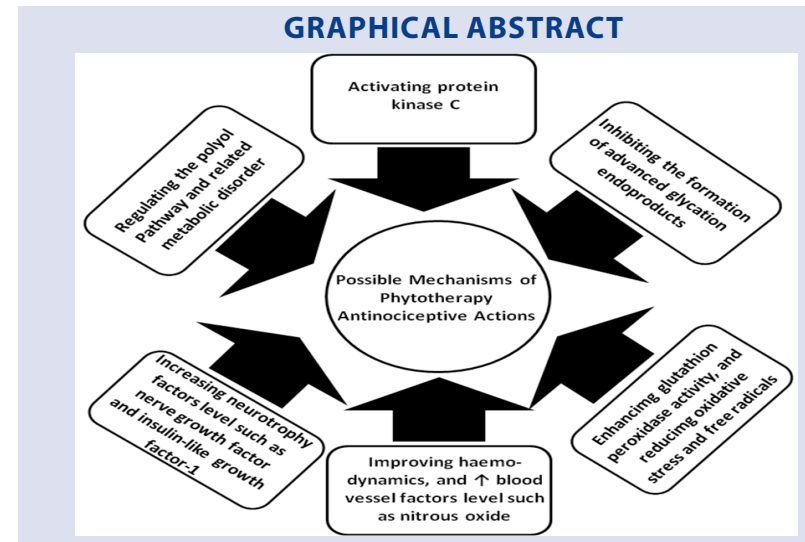

Group Photo: Rawan El-Haj, Dania Shoumar, Rana Alaaeddine, Yousra Fakhro , Natalie Tawil , Farah Shaer, Amani Daher, Natalie Awada, Ali Sabra, Khouloud Atwi, Malak Khaled, Raneem Messi, Nour Abouzaher, Mohamed Houri and Samer Al Jallad: Phytotherapy Junior Research Team, coordinated by Dr. Karim M. Rafaat Associate Professor of Phytochemistry, with a main research focus on the separation and the isolation of medicinally important compounds from natural origin and testing their possible pharmacological and toxicological effects.
- This review discusses neuropathic pain and provides multiple recommendations on how to diagnose and manage this syndrome with a special emphasis on phytotherapy potentials. Phytotherapies, although not yet well studied, offering relatively low-risk options in neuropathic pain patients and having an increasing evidence to be the future of NP management.

\section{AUTHOR PROFILES}

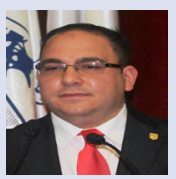

Dr. Karim M. Raafat has completed his PhD from German University in Cairo, New Cairo, Egypt, under the channe system and joint supervision scheme between The German University in Cairo (GUC) and Johann Wolfgang Goethe-University Frankfurt, Germany and postdoctoral studies from Beirut Arab University (BAU) and German University in Cairo. He is a visiting Scientist of Johann Wolfgang Goethe-University, Frankfurt, Germany. He is the Head of Phytochemistry Research Team and Junior Research Team, BAU, Lebanon. He has published more than fifty books, patent, and peer reviewed journal articles and presentations in scientific conferences. He has been honored with several national and international awards in the scientific field and public service.

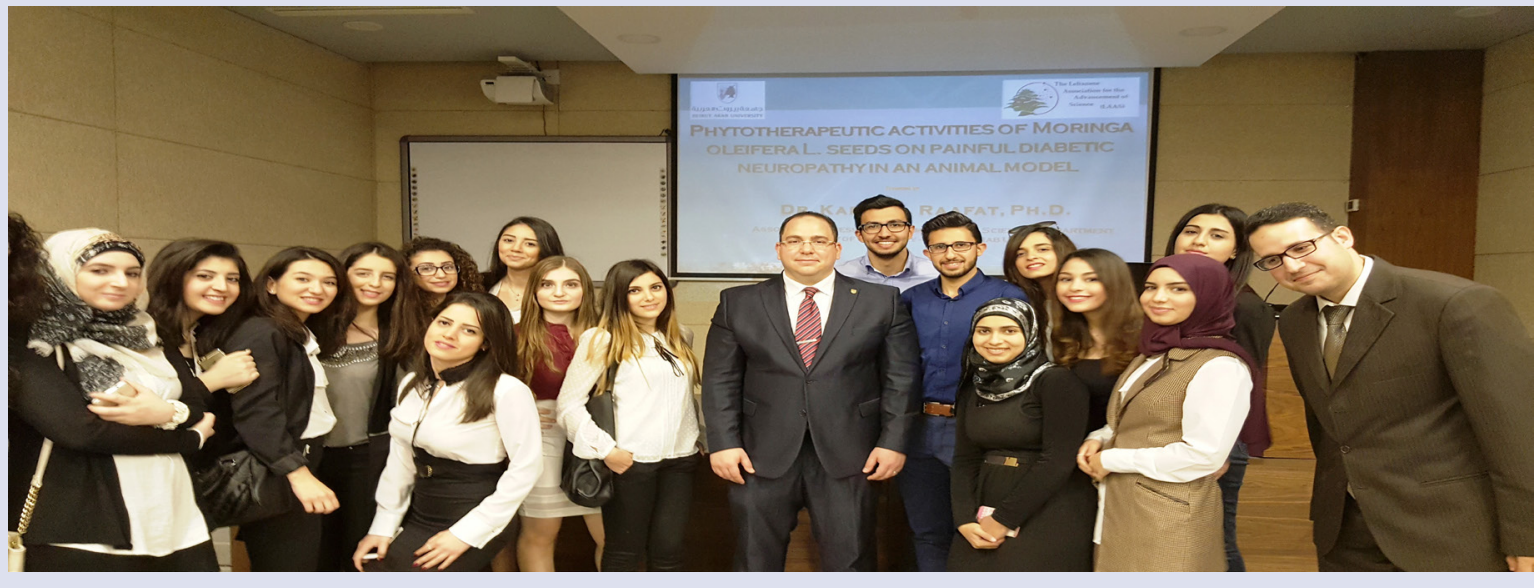

Cite this article: Raafat K, El-Haja R, Shoumara D, Alaaeddinea R, Fakhro Y, Tawil N, Shaera F, Daher A, Awada N, Sabra A, Atwi K, Khaleda M, Messi R, Abouzaher N, Houri M and Al Jallad S. Neuropathic Pain: Literature Review and Recommendations of Potential Phytotherapies. Pharmacog J. 2017;9(4):425-34. 